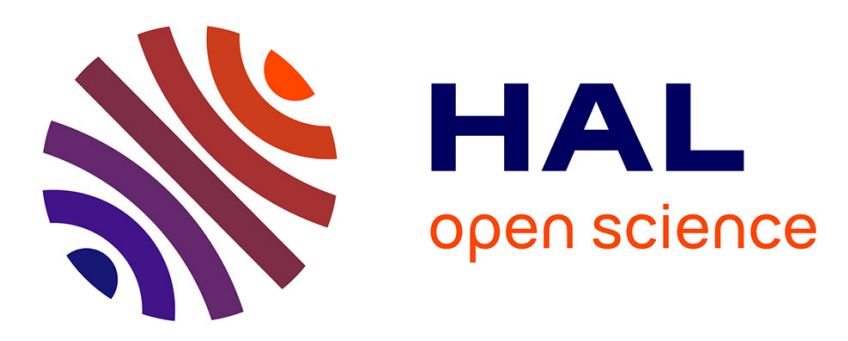

\title{
Second Radiocarbon Intercomparison Program for the Chauvetpont d'Arc Cave, Ardèche, France
}

\author{
A. Quilès, H. Valladas, J-M Geneste, J Clottes, D Baffler, B. Berthier, F \\ Brock, C Bronk Ramsey, E Delqué-Količ, J-P Dumoulin, et al.
}

\section{- To cite this version:}

A. Quilès, H. Valladas, J-M Geneste, J Clottes, D Baffler, et al.. Second Radiocarbon Intercomparison Program for the Chauvetpont d'Arc Cave, Ardèche, France. Radiocarbon, 2014, 56 (2), pp.833-850. 10.2458/56.16940 . hal-02351856

\section{HAL Id: hal-02351856 https://hal.science/hal-02351856}

Submitted on 15 Jun 2021

HAL is a multi-disciplinary open access archive for the deposit and dissemination of scientific research documents, whether they are published or not. The documents may come from teaching and research institutions in France or abroad, or from public or private research centers.
L'archive ouverte pluridisciplinaire HAL, est destinée au dépôt et à la diffusion de documents scientifiques de niveau recherche, publiés ou non, émanant des établissements d'enseignement et de recherche français ou étrangers, des laboratoires publics ou privés. 


\title{
SECOND RADIOCARBON INTERCOMPARISON PROGRAM FOR THE CHAUVET- PONT D'ARC CAVE, ARDĖCHE, FRANCE
}

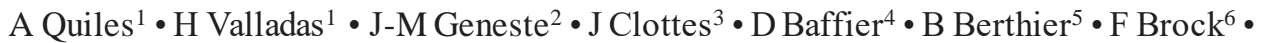

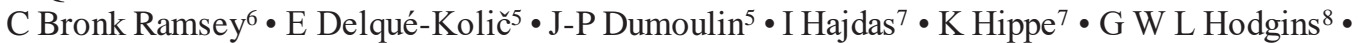

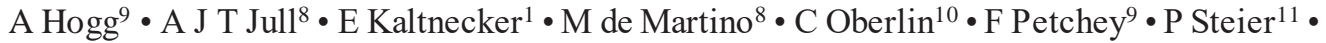 \\ H-A Synal ${ }^{7}$ J van der Plicht ${ }^{12} \bullet$ E M Wild $^{11} \bullet$ A Zazzo $^{13}$
}

ABSTRACT. The Chauvet-Pont d'Arc Cave is one of the most important sites for the study of the earliest manifestations and development of prehistoric art at the beginning of the Upper Paleolithic. Different dating techniques have been performed thus far (AMS ${ }^{14} \mathrm{C}$, U/Th TIMS, ${ }^{36} \mathrm{Cl}$ dating) to model the chronological frame work of this decorated cave. The cave yielded several large charcoal frag ments, which enabled the opportunity for obtaining multiple dates; thus, a First Radiocarbon In tercomparison Program (FIP) was in itiated in 2004 using three charcoal pieces. The FIP de monstrated that those cross-dated samples belonged to a time period associated with the first human occupation. One of the statistical interests of an intercomparis on program is to reduce the uncertainty on the sample age; thus, to further assess the accuracy of the chronological framework, the Second Intercomparison Program (SIP) involving 10 international ${ }^{14} \mathrm{C}$ laboratories was carried out on two pieces of charcoal found inside two hearth structures of the Galerie des Mégacéros. Each laboratory used its own pretreat ment and AMS facilities. In total, 21 and 22 measurements were performed, respectively, which yielded consistent results averaging $\sim 32 \mathrm{ka}$ BP. Two strategies have currently been developed to identify statistical outliers and to deal with them; both lead to quasi-identical calibrated combined densities. Finally, the new results were compared with those of the FIP, leading to the important conclusion that five different samples from at least three different hearth structures give really tightened temporal densities, associated with one short human occupation in the Galerie des Mégacéros.

\section{INTRODUCTION}

The Chauvet-Pont d'Arc Cave (Ardèche, France) was discovered on 18 December 1994 by three speleologists, J-M Chauvet, E Brunel, and C Hillaire. The following year, the French Ministry of Culture put out an International scientific tender, which was won by Jean Clottes' team's proposal, in May 1996. Since 1998, the Chauvet-Pont d'Arc Cave and its famous paintings have been continually studied by this multidisciplinary scientific team, led by J Clottes from 1998 to 2001 (Clottes

1. Laboratoire des Sciences du Climat et de l'Environnement (LSCE/IPSL) CEA-CNRS-UVSQ (UMR 8212), Avenue de la Terrasse, 91198, Gif-Sur-Yvette, France.

2. Centre National de la Préhistoire, Ministère de la culture et de la communication, UMR 5199 CNRS, 38 rue du 26e R.I., 24000 Périgueux, France.

3. 11 rue du Fourcat, 09000 Foix, France.

4. DRAC de Rhône-Alpes, Ministère de la culture et de la communication, CREPS, 07150 Vallon Pont d'Arc, France.

5. Laboratoire de Mesure du Carbone 14, CEA-CNRS-IRD-IRSN-M inistère de la culture et de la communication, bât 450 porte 4E, 91191 Gif-Sur-Yvette, France.

6. Oxford Radiocarbon Accelerator Unit, Research Laboratory for Archaeology, University of Oxford, Dyson Perrins Building, South Parks Road, Oxford OX1 3QY, United Kingdom.

7. Laboratory of Ion Beam Physics, ETH Zürich, Otto-Stern-Weg 5, 8093 Zurich, Switzerland.

8. NSF Arizona AMS Laboratory, University of Arizona, Physics Building, 1118 East Fourth St., Tucson, AZ 85721, USA.

9. Radiocarbon Dating Laboratory, University of Waikato, Gate 9, Hillcrest Road, Hamilton 3240, New Zealand.

10. Centre de datation par le carbone 14, UMR 5138 CNRS, Univ. Claude Bernard Lyon 1, 40 Boulevard Niels Bohr, 69622 Villeurbanne Cedex, France.

11. Faculty of Physics, Isotope Research and Nuclear Physics, VERA-Laboratory, University of Vienna, Waehringer Str. 17, 1090 Vienna, Austria.

12. Center for Isotope Research, Groningen University, Nijenborgh 4, 9747 AG Groningen, the Netherlands; and Faculty of Archaeology, Leiden University, P. O. Box 9515, 2300 RA Leiden, the Netherlands.

13. CNRS - Muséum national d'Histoire naturelle UMR 7209 “Archéozoologie, Archéobotanique: Sociétés, Pratiques et Environnements” USM 303 - Département Écologie et Gestion de la Biodiversité, Case postale 56 (bâtiment d'anatomie comparée), 55, rue Buffon, 75231 Paris Cedex 05, France. 
et al. 1995; Clottes 2001), then by J-M Geneste (2003). This team is currently undertaking research on climatic, geomorphological, paleontological, and biological studies, as well as archaeological rock art context studies, to get a better understanding of the well-preserved and very vivid animal representations (engravings, red and black paintings). In order to get thorough and relevant chronological information on the Paleolithic human occupations in the Chauvet-Pont d'Arc Cave, different dating techniques like accelerator mass spectrometry (AMS) ${ }^{14} \mathrm{C}$ analyses of organic material (Valladas et al. 2004), U/Th by TIMS on speleothems (Genty et al. 2004), and ${ }^{36} \mathrm{Cl}$ on the rock collapse at the entrance of the cave (Sadier et al. 2012) have been carried out and a large spectrum of results is already available. In particular, a broad set of ${ }^{14} \mathrm{C}$ dates has been obtained on ground charcoal, wall drawings, charcoal parietal spots, and animal bones (especially cave bear remains). Dating results from charcoal on the ground show that the human occupation within the cave occurred during two main periods: the first (with $\sim 45{ }^{14} \mathrm{C}$ dates) ranges from 33 to $29.5 \mathrm{ka} \mathrm{BP}$, and the second one, a few millennia later, extends from 27 to $25 \mathrm{ka} \mathrm{BP}$ (with $\sim 15{ }^{14} \mathrm{C}$ dates) (Valladas et al. 2005). Obviously, the Chauvet-Pont d'Arc Cave is one of the most important sites for the study of the earliest manifestations and the de velopment of prehistoric rock art at the beginning of the European Upper Paleolithic (Valladas et al. 2001).

Since the publication of the IntCal09 calibration curve (Reimer et al. 2009), ${ }^{14} \mathrm{C}$ dates older than $26 \mathrm{ka} \mathrm{BP}$ can be calibrated. This improvement has opened new prospects for the study of the chronology of the Chauvet-Pont d'Arc Cave human occupations, and raised the possibility of modeling the dates using a Bayesian approach, which significantly improves chronological precision. The calibration and statistical modeling of the ${ }^{14} \mathrm{C}$ dates are presently in progress. Statistical treatment of all the charcoal on the ground and samples from drawings linked to the first prehistoric occupation suggests that this occupation dates from 37.4 to $33.2 \mathrm{ka} \mathrm{cal} \mathrm{BP} \mathrm{(2 \sigma )} \mathrm{(Quiles} \mathrm{et} \mathrm{al.} \mathrm{2012).}$

The Chauvet-Pont d'Arc Cave yielded archaeological remains including several large charcoal fragments, which offer the valuable opportunity for obtaining multiple dates. To assess the accuracy of the chronological framework, a First Intercomparison Program, involving six ${ }^{14} \mathrm{C}$ laboratories, was initiated in 2004. Successful results of this first program gave an average age of $~ 32 \mathrm{ka} \mathrm{BP}$ for the three pieces of charcoal collected from one archaeological hearth structure in the Galerie des Mégacéros (Cuzange et al. 2007). The comparison of the three samples' calibrated combined ages with all the ground charcoal calibrated dates shows that the cross-dated samples belonged to a time period linked to the first occupation modeled phase, being more probably associated with the oldest part of this occupation phase. This observation suggests two hypotheses:

1) Because those three charcoal pieces were associated with the same archaeological entity, is this observation a coincidence due to the sampling process? Or

2) Does this observation rely on archaeological evidence? It would mean that this part of the modeled phase would correspond to the most probable period for the first human occupation.

In order to answer these questions, a Second Intercomparison Program was carried out for the Chauvet-Pont d'Arc Cave in 2012, under the initiative of the Laboratoire des Sciences du Climat et de l'Environnement and the Chauvet-Pont d'Arc scientific team. Its relevance stems from three factors:

1) Interlaboratory comparisons involving old prehistoric charcoal pieces are rare due to the scarcity of large specimens;

2) Dates obtained by several laboratories on a single archaeological entity make possible the statistical modeling of the results and therefore reduce the associated temporal densities; 
3) The multiplication of modeled densities deduced from different archaeological entities randomly chosen within the Chauvet-Pont d'Arc Cave enables the building of a complex and robust model.

This Second Intercomparison Program was carried out on two pieces of charcoal found inside two different and independent archaeological entities in the Galerie des Mégacéros; they were chosen because of their size. They have been analyzed by the 10 independent international ${ }^{14} \mathrm{C}$ laboratories listed below. Each one used its own chemical pretreatment and AMS facility:

1) Laboratoire des Sciences du Climat et de l'Environnement (Gif-Sur-Yvette, France; GifA);

2) Center for Isotope Research (Groningen, the Netherlands; GrA);

3) Oxford Radiocarbon Accelerator Unit (Oxford, UK; ORAU);

4) Centre de datation par le carbone 14 (Lyon, France; Lyon);

5) Museum National d'Histoire Naturelle (Paris, France; Muse);

6) Laboratoire de Mesure du Carbone 14 (Saclay, France; SacA);

7) NSF Arizona AMS Laboratory (Tucson, USA; AA);

8) VERA Laboratory (Vienna, Austria; VERA);

9) Radiocarbon Dating Laboratory (Waikato, New Zealand; Wk);

10) Laboratory of Ion Beam Physics ETH (Zurich, Switzerland; ETH).

The relationship between the two hearth structures from which the charcoal specimens were sampled and the black paintings is fundamental for the understanding of the Chauvet-Pont d'Arc Cave occupations. As it remains a crucial issue, this point is presently being rigorously examined by the Chauvet-Pont d'Arc scientific team and will be thoroughly discussed in a forthcoming article, which will complement the present one. Here, we closely focus on the radiometric results of the Second Intercomparison Program and on the way to deal with them, using the presented statistical approach. After having analyzed the ${ }^{14} \mathrm{C}$ results, we will develop a strategy to identify statistical outliers and to deal with them. Those new results will then be combined and compared with those of the First Intercomparison Program, to finally model the human occupation phase in the Galerie des Mégacéros of the Chauvet-Pont d'Arc Cave.

\section{MATERIALS AND METHODS}

The Second Intercomparison Program was carried out on two large pieces of charcoal, GC-12-01 and GC-12-04, sampled in March 2012 from two different structures at the lower part of the Galerie des Mégacéros (Figure 1): the first (GC-12-01) comes from a hearth structure located to the right of the footbridge (Figure 1a); and the second, from another charcoal concentration to the left of the footbridge, a few meters forward (Figure 1b). These two pieces of charcoal have been identified as Pinus cf. sylestris/nigra by I Théry (CEPAM, Nice, France); they were big enough to be split into portions weighing between 120 and $250 \mathrm{mg}$ (Figure 2) and sent to the 10 laboratories involved in this program. Each laboratory followed its own chemical pretreatment and used its own AMS facility.

Table 1 reports the chemical protocol applied by each laboratory and the AMS facility used (columns 3 and 4). The 10 laboratories carried out acid-base-acid (ABA) pretreatment even if they have used different acid/base concentrations. The ETH, ORAU, and LSCE laboratories also tested the more aggressive ABOX pretreatment (Bird et al. 1999; Brock et al. 2010; Hajdas et al. 2007), using potassium dichromate in sulfuric acid without precombustion for $\mathrm{ETH}$, and with a $300^{\circ} \mathrm{C}$ and 


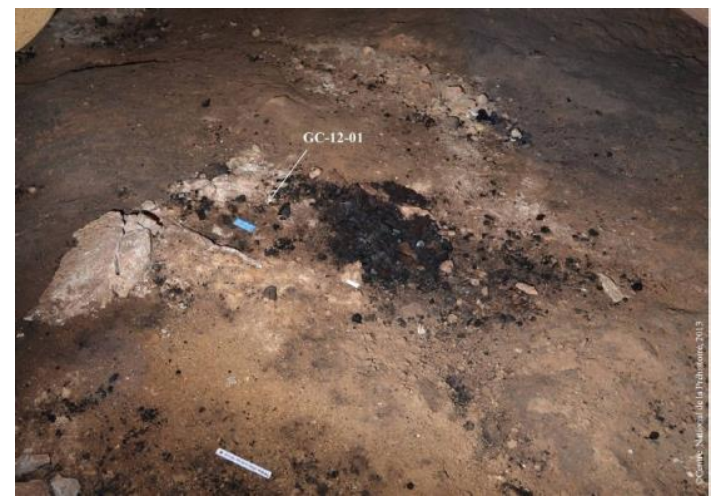

Figure 1 Archaeological hearth structures in the Galerie des Mégacéros, from which GC-12-01 (a) and GC-12-04, GC-40, GC-41, and GC-42 (b) were sampled. C) Centre National de la Préhistoire, France, 2013.

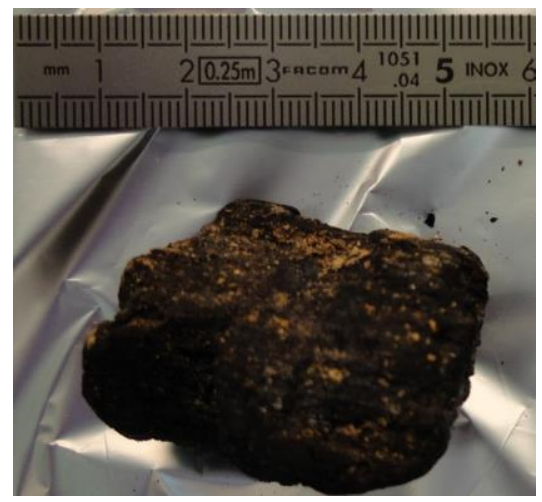

Figure 2 Sample GC-12-04 lifted from an archaeological hearth structure in the Galerie des Mégacéros. CLaboratoire des Sciences du Climat et de l'Environnement, France, 2012.

a $630^{\circ} \mathrm{C}$ precombustion for, respectively, LSCE and ORAU. ETH also tested replacing $\mathrm{HCl}$ with sulfuric acid treatment in the second acid step $\left(2.0 \mathrm{M} \mathrm{H}_{2} \mathrm{SO}_{4} ; \mathrm{ETH}-46133 \mathrm{~b}\right.$ and ETH-46134b). The heterogeneity of the charcoal samples makes some portions more exposed than others. For instance, for GC-12-01, ABA or ABOX treatments have been performed by nine laboratories whereas Oxford had to perform a "mild acid only" (as the charcoal dissolved in the base step and no yield was obtained despite several attempts of their routine ABA treatment); we suspect they got an exposed, and therefore degraded, piece of charcoal. VERA laboratory also dated the humic fractions resulting from the alkaline pretreatment of the two samples as well as the ABA-treated sample GC-12-01. Furthermore, most laboratories performed duplicate samples that give a direct assessment of their repeatability. The Waikato laboratory pretreated and graphitized its samples, including standards associated with the wheel. The unknown samples and standards have then been measured at the University of California Irvine (UCI) laboratory in a single wheel. They apply corrections based on backgrounds and moderns whereas Waikato applied a laboratory correction based on the in-house standards that they use to monitor their repeatability.

\section{RESULTS}

$\delta^{13} \mathrm{C}$, background values, and ${ }^{14} \mathrm{C}$ ages for each measurement are reported in Table 1, columns 5-7. In total, 21 analyses were performed on GC-12-01 and 22 on GC-12-04, on charcoal fractions obtained after at least four different chemical pretreatments (ABA, ABOX, "mild acid only," alkaline fraction, according to the heterogeneity of the charcoal sample) and with seven different AMS facilities. In the Gif, Groningen, Lyon, MNHN, VERA, Saclay, and Zurich laboratories, $\delta^{13} \mathrm{C}$ values were determined during the AMS measurements (they are provided with an uncertainty of $\sim 3 \%$ ), whereas the Oxford, Tucson, and Waikato laboratories performed measurements by mass spectrometry during the combustion process (in italics in Table 1, Column 5).

\section{GC-12-01}

The $21{ }^{14} \mathrm{C}$ dates performed on GC-12-01 range from $32,670 \pm 380$ to $31,120 \pm 180 / 170 \mathrm{BP}$; $\delta^{13} \mathrm{C}$ values ranged from -25.3 to $-19.5 \%$. Those $21{ }^{14} \mathrm{C}$ ages are compatible within a $2 \sigma$ range (Figure 3 ); they are consistent and give an average value of 31,979 $\pm 378 \mathrm{BP}$. We note that ETH-46133-a was performed on a small sample containing $0.3 \mathrm{mg}$ of carbon; consequently, the blank correction and uncertainty are larger than the ones obtained for the other measurements, while remaining compatible with the others. Alkaline fractions were also measured (VERA-5579HS and VERA-5779HS_2) 
Table 1 Results of the Second Intercomparison Programme carried out on the two ground charcoal specimens GC-12-01 and GC-12-04. The pretreatment protocols and AMS facilities used are detailed in columns 3 and $4 . \delta^{13} \mathrm{C}$ are in column 5 (values obtained by mass spectrometry are in italics, others were measured by AMS). Background corrections (in R14 value, with R14 = pMC/103.98) applied to each measurement are reported in column 6. Radiocarbon ages are given in BP (column 7); some laboratories performed multiple dates and their a verage values are calculated (column 8). daverage calculates the dispersion of a single date to the total average value (column 9). d $\sigma$ calculates the dispersion of a single date uncertainty to the uncertainty's minimun (column 10). Maxima of daverage and $d \sigma$ are reported at the bottom of columns 9 and 10 for GC-12-01 and GC-12-04. To identify outliers (WOS), an a priori probability was set to 5\% for each sample's measurements. and the $a$ posteriori outlier probabilities were calculated using OxCal 4.2 R_Combine tool; they are reported column 11 (prior/posterior).

\begin{tabular}{|c|c|c|c|c|c|c|c|c|c|c|c|c|}
\hline \multirow{2}{*}{$\begin{array}{l}\text { Chauvet } \\
\text { sample }\end{array}$} & \multirow[b]{2}{*}{ Sample nr } & \multirow[b]{2}{*}{ Sample (pretreatment) } & \multirow[b]{2}{*}{ AMS facility } & \multirow[b]{2}{*}{$\delta^{13} \mathrm{C}$} & \multirow{2}{*}{$\begin{array}{l}\text { Back- } \\
\text { ground } \\
\text { (R14) }\end{array}$} & \multicolumn{3}{|c|}{${ }^{14} \mathrm{C}$ age $\mathrm{BP}$} & \multirow{2}{*}{$\begin{array}{l}\text { Mean } \\
\text { value/ } \\
\text { lab } \\
\text { (BP) }\end{array}$} & \multirow[b]{2}{*}{$\delta$ ave. } & \multirow[b]{2}{*}{$\delta \sigma$} & \multirow{2}{*}{$\begin{array}{l}\text { Outlier } \\
\text { test }(s) \\
\text { R_Com- } \\
\text { bine }\end{array}$} \\
\hline & & & & & & & $\sigma^{+}$ & $\sigma^{-}$ & & & & \\
\hline \multirow[t]{8}{*}{$\overline{\text { GC-12-01 }}$} & Wk 33807 & $\begin{array}{l}\text { Charcoal(ABA) } \\
\text { Charcoal(ABA) }\end{array}$ & $\begin{array}{r}0.5 \mathrm{MV} \text { NEC - UC Ir- } \\
\text { vine Keck-CCAMS }\end{array}$ & $\begin{array}{l}-24.3 \pm 0.2 \\
-24.3 \pm 0.2\end{array}$ & 0.0019 & $\begin{array}{l}32,160 \\
32,328\end{array}$ & $\begin{array}{l} \pm 278 \\
\pm 400\end{array}$ & $\begin{array}{l}278 \\
400\end{array}$ & 32,244 & $\begin{array}{l}0.56 \\
1.08\end{array}$ & $\begin{array}{l}35.25 \\
55.00\end{array}$ & $\begin{array}{l}5 / 3 \\
5 / 4\end{array}$ \\
\hline & $\begin{array}{l}\text { GrA53780 } \\
\text { GrA53781 }\end{array}$ & $\begin{array}{l}\text { Charcoal(ABA) } \\
\text { Charcoal(ABA) }\end{array}$ & $\begin{array}{ll}\text { HVEE-4130, } & 2.5 \mathrm{MV} \\
\text { HVEE-4130, } & 2.5 \mathrm{MV}\end{array}$ & $\begin{array}{l}-23.6 \\
-23.1\end{array}$ & $\begin{array}{l}0.0022 \\
0.0022\end{array}$ & $\begin{array}{l}31,800 \\
31,710\end{array}$ & $\begin{array}{l} \pm 230 \\
\pm 250\end{array}$ & $\begin{array}{l}210 \\
230\end{array}$ & 31,755 & $\begin{array}{l}0.56 \\
0.85\end{array}$ & $\begin{array}{l}14.29 \\
21.74\end{array}$ & $\begin{array}{l}5 / 3 \\
5 / 5\end{array}$ \\
\hline & ETH-46133 & $\begin{array}{l}\text { a) Charcoal (ABA) small } \\
\text { b) Charcoal }\left(\mathrm{ABOX} \mathrm{H}_{2} \mathrm{SO}_{4}\right) \\
\text { c) Charcoal }\left(\mathrm{ABOXX \textrm {K } _ { 2 }} \mathrm{Cr}_{2} \mathrm{O}_{7}\right) \\
\text { d) Charcoal }(\mathrm{ABA})\end{array}$ & Micadas & $\begin{array}{l}-23.1 \pm 1.1 \\
-23.4 \pm 1.1 \\
-24.2 \pm 1.1 \\
-23.0 \pm 1.2\end{array}$ & $\begin{array}{l}0.0086 \\
0.0019 \\
0.0019 \\
0.0019\end{array}$ & $\begin{array}{l}31,815 \\
31,297 \\
31,619 \\
31,540\end{array}$ & $\begin{array}{l} \pm 855 \\
\pm 227 \\
\pm 238 \\
\pm 234\end{array}$ & $\begin{array}{l}855 \\
227 \\
238 \\
234\end{array}$ & 31,568 & $\begin{array}{l}0.51 \\
2.18 \\
1.14 \\
1.39\end{array}$ & $\begin{array}{l}78.95 \\
20.70 \\
24.37 \\
23.08\end{array}$ & $\begin{array}{l}5 / 2 \\
5 / 87 \\
5 / 11 \\
5 / 22\end{array}$ \\
\hline & $\begin{array}{l}\text { Lyon-8930 (GrA) } \\
\text { Lyon-9299 (SacA } \\
\text { 29721) }\end{array}$ & $\begin{array}{l}\text { Charcoal(ABA) } \\
\text { Charcoal(ABA) }\end{array}$ & $\begin{array}{l}\text { HVEE-4130, } 2.5 \mathrm{MV} \\
\text { 3MV NEC Pelletron } \\
\text { Artemis }\end{array}$ & $\begin{array}{r}- \\
-22.8\end{array}$ & $\begin{array}{l}0.0022 \\
0.0073\end{array}$ & $\begin{array}{l}31,120 \\
31,360\end{array}$ & $\begin{array}{l} \pm 180 \\
\pm 860\end{array}$ & $\begin{array}{l}170 \\
860\end{array}$ & 31,240 & $\begin{array}{l}2.76 \\
1.97\end{array}$ & $\begin{array}{r}0.00 \\
79.07\end{array}$ & $\begin{array}{l}5 / 100 \\
5 / 3\end{array}$ \\
\hline & $\begin{array}{l}\text { SacA } 28829 \\
\text { SacA } 29314\end{array}$ & $\begin{array}{l}\text { Charcoal(ABA) } \\
\text { Charcoal(ABA) }\end{array}$ & $\begin{array}{l}\text { 3MV NEC Pelletron } \\
\text { Artemis }\end{array}$ & $\begin{array}{l}-22.4 \\
-21.1\end{array}$ & $\begin{array}{l}0.0024 \\
0.0024\end{array}$ & $\begin{array}{l}31,940 \\
32,380\end{array}$ & $\begin{array}{l} \pm 390 \\
\pm 410\end{array}$ & $\begin{array}{l}390 \\
410\end{array}$ & 32,160 & $\begin{array}{l}0.12 \\
1.24\end{array}$ & $\begin{array}{l}53.85 \\
56.10\end{array}$ & $\begin{array}{l}5 / 2 \\
5 / 5\end{array}$ \\
\hline & OxA-26572 & Charcoal(“mild acid only") & 3MV HVEE & -23.6 & - & 32,500 & \pm 310 & 310 & - & 1.60 & 41.94 & $5 / 14$ \\
\hline & $\begin{array}{l}\text { VERA-5779HS } \\
\text { VERA-5779_2 } \\
\text { VERA-5779HS_2 }\end{array}$ & $\begin{array}{l}\text { Alkaline fraction } \\
\text { Charcoal(ABA) } \\
\text { Alkaline fraction (extracted } \\
\text { from VERA-5579_2) }\end{array}$ & 3MV NEC Pelletron & $\begin{array}{l}-25.3 \pm 1.1 \\
-23.7 \pm 1.6 \\
-19.5 \pm 3.5\end{array}$ & $\begin{array}{l}0.0015 \\
0.0012 \\
0.0012\end{array}$ & $\begin{array}{l}32,180 \\
32,158 \\
31,547\end{array}$ & $\begin{array}{l} \pm 444 \\
\pm 276 \\
\pm 264\end{array}$ & $\begin{array}{l}421 \\
266 \\
255\end{array}$ & 31,962 & $\begin{array}{l}0.63 \\
0.56 \\
1.37\end{array}$ & $\begin{array}{l}59.46 \\
34.78 \\
31.82\end{array}$ & $\begin{array}{l}5 / 2 \\
5 / 3 \\
5 / 14\end{array}$ \\
\hline & $\begin{array}{l}\text { GifA 13014 (SacA } \\
\text { 32303) } \\
\text { GifA 13015 (SacA } \\
\text { 32304) } \\
\text { GifA 13019 (SacA } \\
\text { 32308) }\end{array}$ & $\begin{array}{l}\text { Charcoal(ABA) } \\
\text { Charcoal(ABA) } \\
\text { Charcoal(ABOX) }\end{array}$ & $\begin{array}{l}\text { 3MV NEC Pelletron } \\
\text { Artemis }\end{array}$ & $\begin{array}{l}-23.1 \\
-22.8 \\
-24.2\end{array}$ & $\begin{array}{l}0.0025 \\
0.0025 \\
0.0025\end{array}$ & $\begin{array}{l}32,640 \\
32,330 \\
32,670\end{array}$ & $\begin{array}{l} \pm 400 \\
\pm 370 \\
\pm 380\end{array}$ & $\begin{array}{l}400 \\
370 \\
380\end{array}$ & 32,547 & $\begin{array}{l}2.03 \\
1.09 \\
2.12\end{array}$ & $\begin{array}{l}55.00 \\
51.35 \\
52.63\end{array}$ & $\begin{array}{l}5 / 22 \\
5 / 4 \\
5 / 33\end{array}$ \\
\hline
\end{tabular}


Table (Continued)

\begin{tabular}{|c|c|c|c|c|c|c|c|c|c|c|c|c|}
\hline \multirow{2}{*}{$\begin{array}{l}\text { Chauvet } \\
\text { sample }\end{array}$} & \multirow[b]{2}{*}{ Sample $\mathrm{nr}$} & \multirow[b]{2}{*}{ Sample (pretreatment) } & \multirow[b]{2}{*}{ AMS facility } & \multirow[b]{2}{*}{$\delta^{13} \mathrm{C}$} & \multirow{2}{*}{$\begin{array}{l}\text { Back- } \\
\text { ground } \\
\text { (R14) }\end{array}$} & \multicolumn{3}{|c|}{${ }^{14} \mathrm{C}$ age $\mathrm{BP}$} & \multirow{2}{*}{$\begin{array}{l}\text { Mean } \\
\text { value/ } \\
\text { lab } \\
(\mathrm{BP})\end{array}$} & \multirow[b]{2}{*}{$\delta$ ave. } & \multirow[b]{2}{*}{$\delta \sigma$} & \multirow{2}{*}{$\begin{array}{l}\text { Outlier } \\
\text { test }(s) \\
\text { R_Com- } \\
\text { bine }\end{array}$} \\
\hline & & & & & & & $\sigma^{+}$ & $\sigma^{-}$ & & & & \\
\hline & AA 98841 & Charcoal(ABA) & 3MV NEC & -23.6 & 0.0024 & 32,170 & \pm 470 & 470 & - & 0.59 & 61.70 & $5 / 2$ \\
\hline & $\begin{array}{l}\text { Muse 240 (SacA } \\
\quad 31533)\end{array}$ & Charcoal & $\begin{array}{l}\text { 3MV NEC Pelletron } \\
\text { Artemis }\end{array}$ & -24.2 & 0.0029 & 32,290 & \pm 510 & 510 & - & 0.96 & 64.71 & $5 / 3$ \\
\hline & & & & & Average & 31,979 & \pm 378 & - & $\max :$ & 2.76 & 79.07 & \\
\hline \multirow[t]{11}{*}{ GC-12-04 } & Wk 33808 & $\begin{array}{l}\text { Charcoal(ABA) } \\
\text { Charcoal(ABA) }\end{array}$ & $\begin{array}{l}\text { 0.5MV NEC -UC Ir- } \\
\text { vine Keck-CAMS }\end{array}$ & $\begin{array}{l}-22.5 \pm 0.2 \\
-22.5 \pm 0.2\end{array}$ & 0.0019 & $\begin{array}{l}32,131 \\
32,284\end{array}$ & $\begin{array}{l} \pm 272 \\
\pm 398\end{array}$ & $\begin{array}{l}272 \\
398\end{array}$ & 32,208 & $\begin{array}{l}0.22 \\
0.69\end{array}$ & $\begin{array}{l}12.50 \\
40.20\end{array}$ & $\begin{array}{l}5 / 2 \\
5 / 3\end{array}$ \\
\hline & $\begin{array}{l}\text { GrA53609 } \\
\text { GrA53610 }\end{array}$ & $\begin{array}{l}\text { Charcoal(ABA) } \\
\text { Charcoal(ABA) }\end{array}$ & $\begin{array}{ll}\text { HVEE-4130. } & 2.5 \mathrm{MV} \\
\text { HVEE-4130. } & 2.5 \mathrm{MV}\end{array}$ & $\begin{array}{l}-23.2 \\
-23.3\end{array}$ & $\begin{array}{l}0.0022 \\
0.0022\end{array}$ & $\begin{array}{l}32,810 \\
32,910\end{array}$ & $\begin{array}{l} \pm 320 \\
\pm 320\end{array}$ & $\begin{array}{l}280 \\
280\end{array}$ & 32,860 & $\begin{array}{l}2.28 \\
2.58\end{array}$ & $\begin{array}{l}15.00 \\
15.00\end{array}$ & $\begin{array}{l}5 / 83 \\
5 / 96\end{array}$ \\
\hline & ETH-46134 & $\begin{array}{l}\text { a) Charcoal (ABA) } \\
\text { b) Charcoal }\left(\mathrm{ABOX} \mathrm{H}_{2} \mathrm{SO}_{4}\right) \\
\text { c) Charcoal }\left(\mathrm{ABOX} \mathrm{K} \mathrm{Cr}_{2} \mathrm{O}_{7}\right) \\
\text { d) Charcoal }(\mathrm{ABA})\end{array}$ & Micadas & $\begin{array}{l}-22.1 \pm 1.1 \\
-24.5 \pm 1.1 \\
-23.7 \pm 1.1 \\
-26.6 \pm 1.1\end{array}$ & $\begin{array}{l}0.0032 \\
0.0019 \\
0.0019 \\
0.0019\end{array}$ & $\begin{array}{l}31,875 \\
31,864 \\
31,663 \\
31,886\end{array}$ & $\begin{array}{l} \pm 265 \\
\pm 245 \\
\pm 238 \\
\pm 247\end{array}$ & $\begin{array}{l}265 \\
245 \\
238 \\
247\end{array}$ & 31,822 & $\begin{array}{l}0.58 \\
0.62 \\
1.26 \\
0.55\end{array}$ & $\begin{array}{r}10.19 \\
2.86 \\
0.00 \\
3.64\end{array}$ & $\begin{array}{l}5 / 2 \\
5 / 3 \\
5 / 10 \\
5 / 2\end{array}$ \\
\hline & $\begin{array}{l}\text { Lyon-8931 (GrA) } \\
\text { Lyon-9300 (SacA } \\
\text { 29722) }\end{array}$ & $\begin{array}{l}\text { Charcoal(ABA) } \\
\text { Charcoal(ABA) }\end{array}$ & $\begin{array}{l}\text { HVEE-4130. } 2.5 \mathrm{MV} \\
\text { 3MV NEC Pelletron } \\
\text { Artemis }\end{array}$ & $\begin{array}{l}- \\
-23.4\end{array}$ & $\begin{array}{l}0.0022 \\
0.0073\end{array}$ & $\begin{array}{l}31,940 \\
32,430\end{array}$ & $\begin{array}{l} \pm 200 \\
\pm 980\end{array}$ & $\begin{array}{l}180 \\
980\end{array}$ & 32,185 & $\begin{array}{l}0.38 \\
1.14\end{array}$ & $\begin{array}{l}19.00 \\
75.71\end{array}$ & $\begin{array}{l}5 / 2 \\
5 / 2\end{array}$ \\
\hline & $\begin{array}{l}\text { SacA } 28830 \\
\text { SacA } 29315\end{array}$ & $\begin{array}{l}\text { Charcoal(ABA) } \\
\text { Charcoal(ABA) }\end{array}$ & $\begin{array}{l}\text { 3MV NEC Pelletron } \\
\text { Artemis }\end{array}$ & $\begin{array}{l}-22.0 \\
-21.2\end{array}$ & $\begin{array}{l}0.0024 \\
0.0024\end{array}$ & $\begin{array}{l}32,060 \\
32,290\end{array}$ & $\begin{array}{l} \pm 400 \\
\pm 410\end{array}$ & $\begin{array}{l}400 \\
410\end{array}$ & 32,175 & $\begin{array}{l}0.00 \\
0.71\end{array}$ & $\begin{array}{l}40.50 \\
41.95\end{array}$ & $\begin{array}{l}5 / 2 \\
5 / 3\end{array}$ \\
\hline & $\begin{array}{l}\text { OxA } 26473 \\
\text { OxA } 26485 \\
\text { OxA } 26645\end{array}$ & $\begin{array}{l}\text { Charcoal(ABA) } \\
\text { Charcoal(ABA) } \\
\text { Charcoal(ABOX) }\end{array}$ & 3MV HVEE & $\begin{array}{l}-22.8 \\
-23.4 \\
-22.2\end{array}$ & - & $\begin{array}{l}31,900 \\
31,600 \\
31,910\end{array}$ & $\begin{array}{l} \pm 280 \\
\pm 450 \\
\pm 250\end{array}$ & $\begin{array}{l}280 \\
450 \\
250\end{array}$ & 31,803 & $\begin{array}{l}0.50 \\
1.46 \\
0.47\end{array}$ & $\begin{array}{r}15.00 \\
47.11 \\
4.80\end{array}$ & $\begin{array}{l}5 / 2 \\
5 / 4 \\
5 / 2\end{array}$ \\
\hline & $\begin{array}{l}\text { VERA-5780HS } \\
\text { VERA-5780HS_2 }\end{array}$ & $\begin{array}{l}\text { Alkaline fraction } \\
\text { Alkaline fraction }\end{array}$ & 3MV NEC Pelletron & $\begin{array}{l}-24.8 \pm 0.8 \\
-28.1 \pm 2.0\end{array}$ & $\begin{array}{l}0.0015 \\
0.0012\end{array}$ & $\begin{array}{l}32,660 \\
31,759\end{array}$ & $\begin{array}{l} \pm 454 \\
\pm 244\end{array}$ & $\begin{array}{l}430 \\
237\end{array}$ & 32,210 & $\begin{array}{l}1.83 \\
0.95\end{array}$ & $\begin{array}{r}47.58 \\
2.46\end{array}$ & $\begin{array}{l}5 / 11 \\
5 / 4\end{array}$ \\
\hline & $\begin{array}{l}\text { GifA 13016 (SacA } \\
\text { 32305) } \\
\text { GifA 13017 (SacA } \\
\text { 32306) } \\
\text { GifA 13020 (SacA } \\
\text { 32309) }\end{array}$ & $\begin{array}{l}\text { Charcoal(ABA) } \\
\text { Charcoal(ABA) } \\
\text { Charcoal(ABOX) }\end{array}$ & $\begin{array}{l}\text { 3MV NEC Pelletron } \\
\text { Artemis }\end{array}$ & $\begin{array}{l}-23.1 \\
-22.8 \\
-24.2\end{array}$ & $\begin{array}{l}0.0025 \\
0.0025 \\
0.0025\end{array}$ & $\begin{array}{l}32,560 \\
32,600 \\
32,630\end{array}$ & $\begin{array}{l} \pm 500 \\
\pm 390 \\
\pm 390\end{array}$ & $\begin{array}{l}500 \\
390 \\
390\end{array}$ & 32,597 & $\begin{array}{l}1.53 \\
1.65 \\
1.74\end{array}$ & $\begin{array}{l}52.40 \\
38.97 \\
38.97\end{array}$ & $\begin{array}{l}5 / 5 \\
5 / 14 \\
5 / 19\end{array}$ \\
\hline & AA 98842 & Charcoal(ABA) & 3MV NEC Pelletron & -23.3 & 0.0024 & 29,900 & \pm 1000 & 1000 & - & 7.23 & 76.20 & $5 / 29$ \\
\hline & $\begin{array}{l}\text { Muse 241 (SacA } \\
\text { 31534) }\end{array}$ & Charcoal(ABA) & $\begin{array}{l}\text { 3MV NEC Pelletron } \\
\text { Artemis }\end{array}$ & -27.9 & 0.0029 & 31,680 & \pm 460 & 460 & - & 1.20 & 48.26 & $5 / 3$ \\
\hline & & & & & Average & 32,061 & $\pm \mathbf{3 7 3}$ & - & $\max :$ & 7.23 & 76.20 & \\
\hline
\end{tabular}


and are in the same ranges as those measured on the purified charcoal samples; in particular, VERA5779_2 (purified charcoal specimen) gave 32,158 $\pm 276 / 266$ BP and its alkaline fraction 31,547 \pm 264/255 BP (VERA-5779HS_2). Such an agreement between both fractions shows that this sample was not contaminated by modern carbon (Batten et al. 1986).

\section{GC-12-04}

Some 22 measurements were performed by the 10 laboratories on $\mathrm{GC}-12-04 ;{ }^{14} \mathrm{C}$ ages all fall within the $2 \sigma$ range (Figure 4 ) and extend from $32,910 \pm 320 / 280$ to $29,900 \pm 1000 \mathrm{BP}$. The $\delta^{13} \mathrm{C}$ values extend from -28.1 to $-22.2 \%$. As shown in Figure 4, those 22 measurements are compatible with a $2 \sigma$ range and yield an average value of 32,061 $\pm 373 \mathrm{BP}$. Sample AA 98842 seems younger but much of the sample dissolved during the treatment and ${ }^{14} \mathrm{C}$ measurement was performed on only $0.23 \mathrm{mg}$ of carbon, thus the larger uncertainty; howe ver, it remains compatible with remaining results within $2 \sigma$. As for GC-12-01, we note that both the alkaline fraction and various pretreatment protocols lead to compatible results, and so the GC-12-04 sample has not been contaminated.

\section{Conclusions}

In terms of chemical pretreatment, it is truly informative to observe that the four applied pretreatments lead to consistent results, despite the aggressiveness gradient of different agents involved. It allows us to conclude that no modern or extraneous carbon has contaminated those charcoal pieces and that the classical ABA pretreatment could safely be used since the Chauvet-Pont d'Arc Cave's environment preserves charcoal well.

In terms of ${ }^{14} \mathrm{C}$ measurements, we observe that average values per lab for GC-12-01 and GC-12-04 are close (Table 1, column 8) and that maximal dispersion between individual measurements and the global average value (calculated with the 21 and 22 results) is less than 3\%, except AA 98842 $(29,900 \pm 1000 \mathrm{BP})$ which is $7 \%$ ( $\delta$ average are reported Table 1 , column 9$)$. Thus, analytical reproducibility is confirmed whatever the pretreatment protocol and AMS facility.

In term of variations in age uncertainty, we report the variability of the uncertainty $\sigma_{i}$ to the uncertainty's minimum $\sigma_{\text {min }}$ obtained, for each sample (Table 1, column 10):

$$
\delta \sigma=a b s \frac{\left(\sigma_{i}-\min \left(\sigma_{1}: \sigma_{\mathrm{N}}\right)\right.}{\sigma_{i}} * 100
$$

$\delta \sigma$ can grow up to $79.07 \%$ for GC-12-01 (Lyon 9299/SacA 29721) and 76.20\% for GC-12-04 (AA 9842). Such variability in age uncertainty can be explained both by the fact that these ages are close to the detection limit of the method, resulting in a poorer counting statistics, and by different estimations of the variability of the background, at different laboratories.

As explained by Scott (2003), random variation makes the chance of outliers to be roughly $1 / 20$; that is why we expected to get 1 outlier in a set of 20 results. First, to deal with outliers, we have manually looked for ${ }^{14} \mathrm{C}$ measurements that were not compatible with the average value with a $2 \sigma$ range. No such ${ }^{14} \mathrm{C}$ outlier was detected for GC-12-01 and GC-12-04. Then, we used the classical $\chi^{2}$ test to determine if we needed or not to go further in outlier detection. These two tests failed and we obtained for GC-12-01 a value of 48.9 (with an acceptance region of 31.4 for a significance level of $5 \%(31.4,5 \%))$, and for GC-12-04, a value of $34.1(32.7,5 \%)$. 


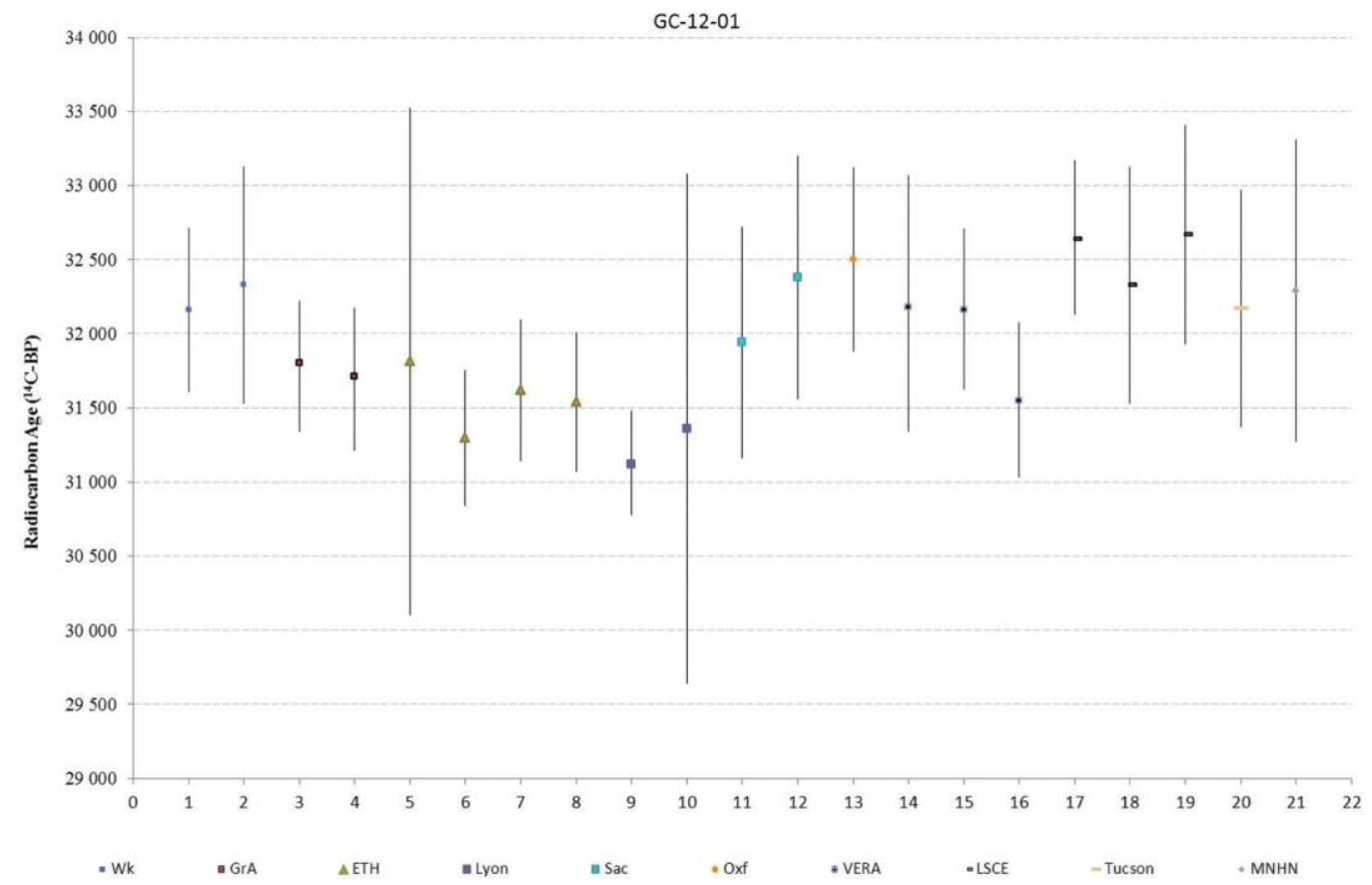

Figure 3 Radiocarbon results obtained for GC-12-01, with a $2 \sigma$ range. The 10 laboratories performed 21 measurements, using their own che mical pretreat ment (A BA, ABOX, "mild acid only," alkaline fraction) and AMS facility (seven different ones). They range from $32,670 \pm 380$ to $31,120 \pm 180 / 170 \mathrm{BP}$ with an average value of $31,979 \pm 378 \mathrm{BP}$; all are co mpatible with a $2 \sigma$ range. Note that the large uncertainly of one ETH analysis is due to the small amount of C used (see text).

\section{INTERPRETATION}

\section{Outlier Detection-Combined Results}

${ }^{14} \mathrm{C}$ ages were calibrated using the OxCal v 4.2 software (Bronk Ramsey 2009a) and the IntCal09 calibration curve (Reimer et al. 2009). To perform a more objective rejection, we applied Bayesian statistical methods to identify outliers in a model averaging approach. The level at which we have to reject or not samples requires analyzing the representativeness of the dated samples related to the timing of the event to which they refer. Usually, random variation of the method as well as variability of the samples' representativeness make the measurements likely to be spurious. Nonetheless, in our case, the two sets of measurements come from two independent pieces of charcoal (GC-12-01 and GC-12-04), so we consider that all measurements performed on the same charcoal specimen are necessarily of the same age. In this case, to identify outliers, we do not have to deal with the samples' representativeness related to a precise archaeological event. The only difficulty would be in individual ${ }^{14} \mathrm{C}$ measurements, which might be at fault.

GC-12-01

We first performed an outlier test of type "s" using OxCal v 4.2 (Bronk Ramsey 2009b), and we chose a normal distribution law as Outlier_Model: "SSimple", $N(0,2), 0$, “ $s$ ". We postulated for all samples a 5\% a priori probability of how likely these individual measurements are to be spurious, in view of the $1 / 20$ chance to be outliers due to random variation of the method. Then, we combined the 21 dates to get a unique age density (Figure 5a). This weighted outlier strategy (WOS) allows for down-weighting those measurements with lower acceptance criteria that are the least consistent. 
GC-12-04

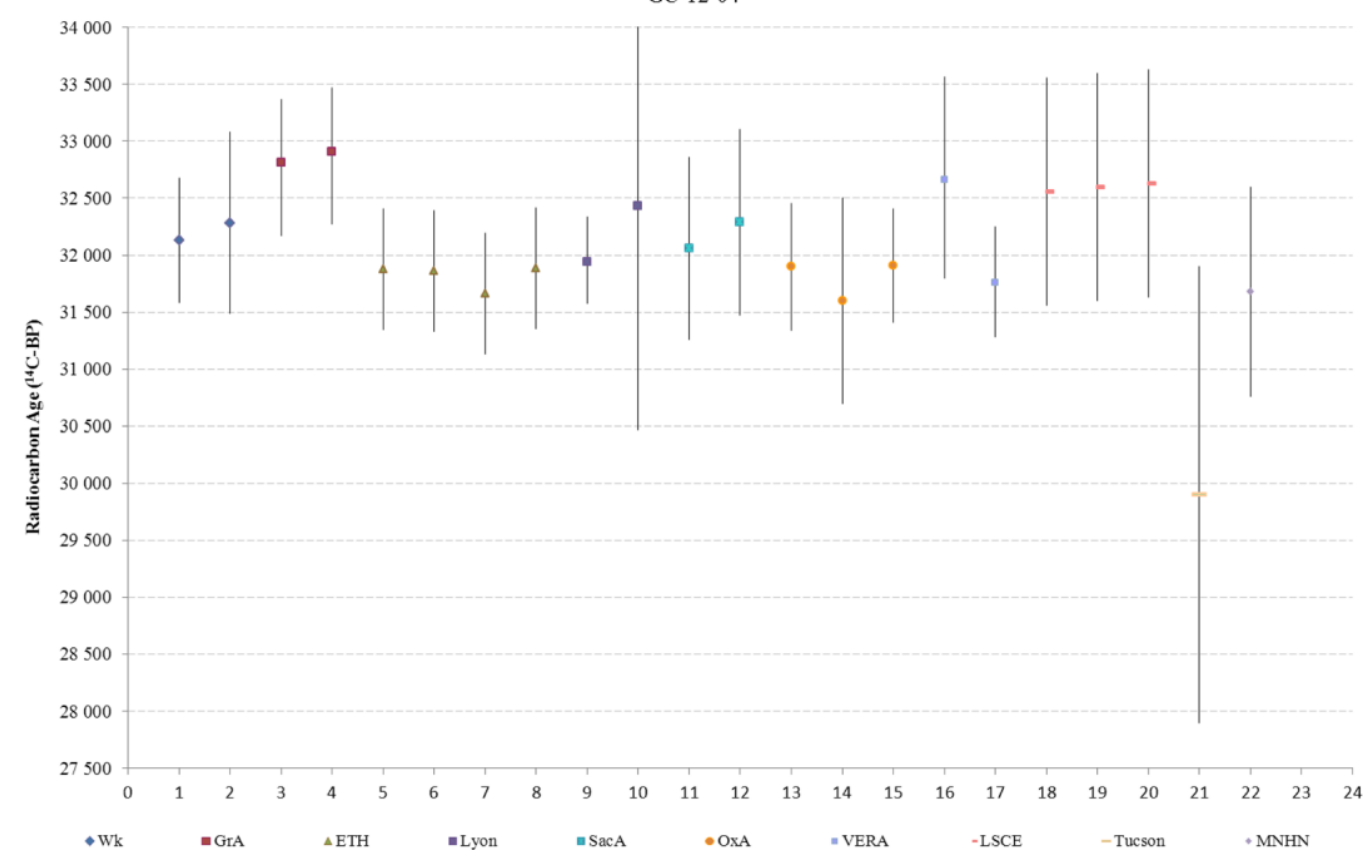

Figure $4{ }^{14} \mathrm{C}$ results obtained for $\mathrm{GC}-12-04$, with a $2 \sigma$ range. The 10 laboratories performed 22 measurements, using their own chemical pretreatment (ABA, ABOX, alkaline fraction) and AMS facility (seven different ones). They range from $32,910 \pm 320 / 280$ to $29,900 \pm 1000 \mathrm{BP}$, with an average value of $32,061 \pm 373 \mathrm{BP}$. Note that the large uncertainty of the AA analysis is due to the small amount of $\mathrm{C}$ used (see text).

Thereby, the deduced combined age remains more influenced by densities associated with a weak outlier probability than to ones reaching the highest probabilities to be outliers. A posteriori outlier probabilities calculated for each measurement are reported in Table 1, column 11. We observe that Lyon-8930 is rejected with an a posteriori outlier probability of 100\%, and the deduced R_Combine age is $31,843 \pm 67 \mathrm{BP}(36,782-36,278 \mathrm{cal} \mathrm{BP}, 2 \sigma)$.

Secondly, we tested a rejected outlier strategy (ROS) to model our results by excluding one after the other those samples that get the highest a posteriori probability to be outliers. Then, we ran the model until obtaining a validated model that passed the $\chi^{2}$ test. For GC-12-01, this outlier test is validated (24.2(5\% 28.9)) if Lyon-8930 and ETH_46133b are rejected. In that case, the R_Combine age is $32,003 \pm 76 \mathrm{BP}(36,766-36,324 \mathrm{cal} \mathrm{BP}, 2 \sigma)$.

GC-12-04

We performed the same outlier tests on GC-12-04 measurements (Figure 5b). Using the WOS, we weighted the measurements with an a priori outlier probability of $5 \%$ for each of them and combined the 22 measurements. A posteriori outlier probabilities obtained so far are summarized in Table 1, column 11. As a result, the model calculated a R_Combine age of 32,078 \pm 68 BP $(36,767-36,325$ cal BP, 95.4\%). Using the ROS, only GrA 53610 was rejected and the resulting model passes the $\chi^{2}$ test $(26.1(31.4,5 \%))$; the R_Combine age is $32,033 \pm 69 \mathrm{BP}(36,776-36,346 \mathrm{cal} \mathrm{BP}, 95.4 \%)$. 
(a)

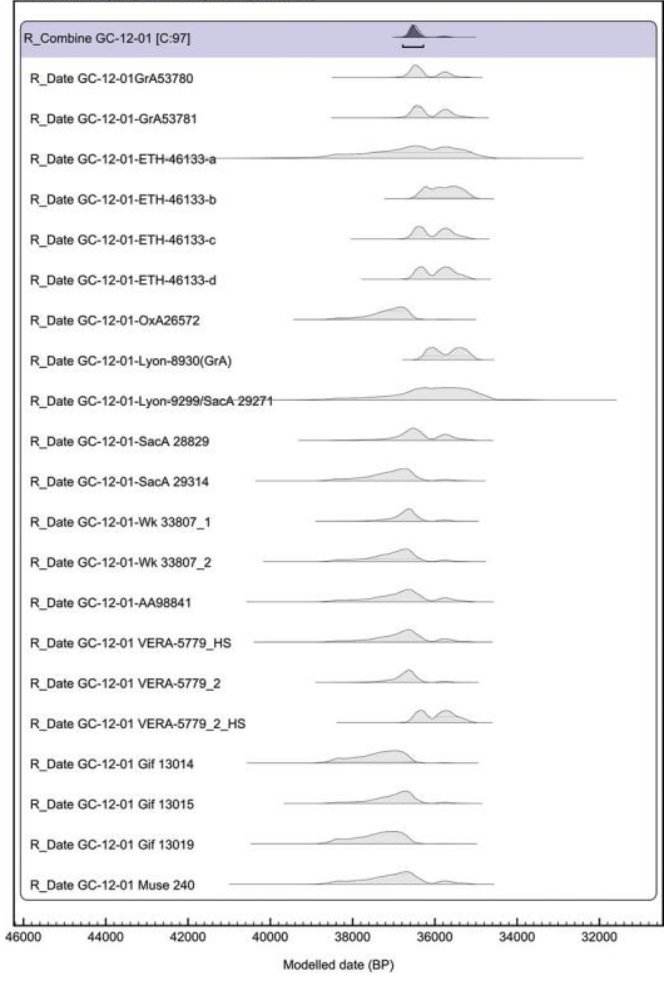

(b)

Figure 5 Outlier tests ("s") were performed on the two sets of measurements done on GC-12-01 (Figure 5a) and GC-12-04 (Figure 5b). An outlier prior probability was set to $5 \%$ for each measure ment; calculated a posteriori probabilities are reported in Table 1. This model calculates a combined age density by sample.

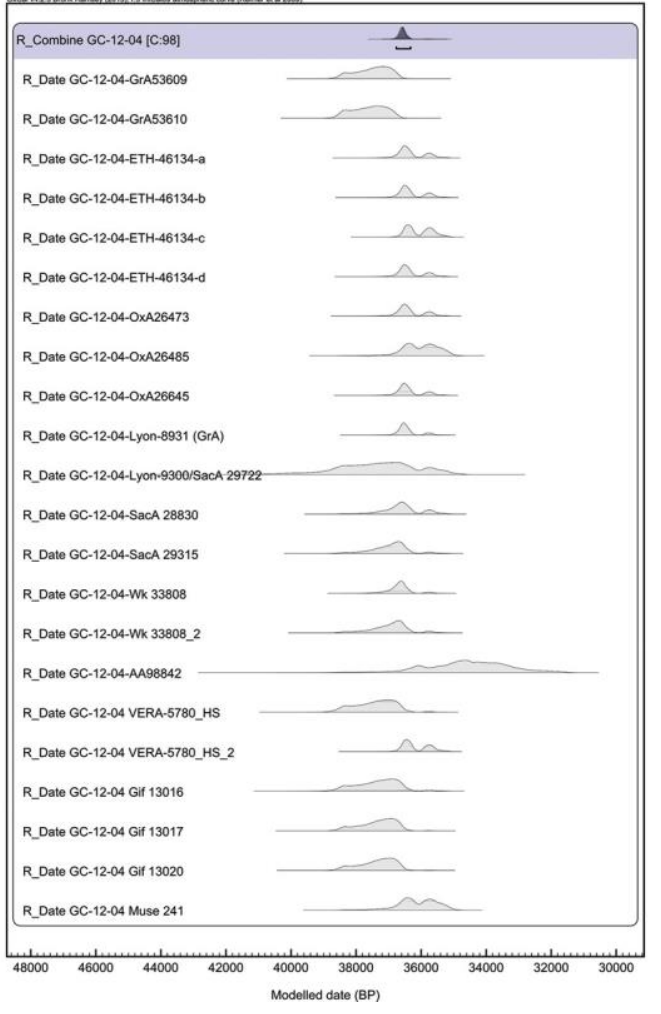




\section{Modeling of the First Intercomparison Results}

The new combined results for GC-12-01 and GC-12-04 might now be compared with the three previous ones GC-40, GC-41, and GC-42, obtained from the First Intercomparison Program. They had been sampled in an archaeological entity in the Galerie des Mégacéros (Figure 1b, Cuzange et al. 2007). Since publication of the first program's results, new measurements were performed on those three samples by both the LSCE and LMC14 laboratories. These additional results are reported in Table 2. They were integrated in the statistical analyses and finally, respectively, 16, 15, and 11 analyses have been carried out on GC-40, GC-41, and GC-42. Figure 6 reports the ${ }^{14} \mathrm{C}$ ages obtained for GC-40 (in blue), GC-41 (in red), and GC-42 (in blue). The three average values are, respectively, $32,034 \pm 324,31,580 \pm 297$, and 31,802 \pm 335 BP. GrA 27040 (GC-40), GrA27316 (GC-41), and GrA 27052 (GC-42) are not consistent with the average value within $2 \sigma$ and are clearly outliers, so they were rejected from the modeling. As previously, the two same outlier tests (WOS and ROS) have been performed on those three sets of results, in order to compare them to the GC-12-01 and GC-12-04 results.

For GC-40, the WOS leads to the a posteriori outlier probabilities reported in Table 2, column 10. GrA 27646 gives an a posteriori probability of $100 \%$, and six samples obtained a value higher than $5 \%$. The deduced R_Combine age is 32,087 \pm 69 BP (36,877-36,420 cal BP, 95.4\%). Following the ROS, GrA 27046 has to be rejected to find a validated model that passes the $\chi^{2}$ test; the deduced R_Combine age is $32,156 \pm 72 \mathrm{BP}(36,866-36,429 \mathrm{cal} \mathrm{BP}, 95.4 \%)$.

Results for GC-41 and GC-42 are gathered in Table 2. WOS leads to a R_Combine age of 31,828 $\pm 70 \mathrm{BP}(36,724-35,719 \mathrm{cal} \mathrm{BP}, 95.4 \%)$ for GC-41 and 31,832 $\pm 81 \mathrm{BP}(36,641-35,599 \mathrm{cal} \mathrm{BP}$, 95.4\%) for GC-42. ROS leads to exclude GifA 70055 and to compute a R_Combine age of 31,875 \pm 72 BP $(36,700-35,705 \mathrm{cal} \mathrm{BP}, 95.4 \%)$ for GC-41. For GC-42, OxA 13976 has to be excluded; the R_Combine age is $31,782 \pm 97 \mathrm{BP}(36,652-35,610 \mathrm{cal} \mathrm{BP}, 95.4 \%)$. We note that GC-41 is very close to passing the $\chi^{2}$ test $(21.787(21.0,5 \%))$, and we prefer to conserve a set of 13 values rather than rejecting one more value. The $\chi^{2}$ test is validated for GC- 42 .

\section{Modeled Results}

To set up a model and to test its robustness, we can compare the results obtained from both outlier strategies (Table 3). An average difference of $-36{ }^{14} \mathrm{C}$ yr is calculated between the two combined ${ }^{14} \mathrm{C}$ ages deduced respectively from WOS and ROS calculations. To investigate if this offset could be significant, we calibrated the deduced combined densities obtained from the WOS and ROS tests. The calibrated densities are summarized in Table 3 and Figure 7 (WOS densities in green and ROS ones in red) and we deduce that both WOS and ROS strategies lead to two quasi-identical densities, despite the offset between the combined ${ }^{14} \mathrm{C}$ ages. This means that whate ver the strategy, the combined calendar densities are the same, which demonstrates the robustness of the modeling. As we had no chemical or physical arguments for rejecting samples, we decided to use the results of the WOS to model our dates.

On average, ${ }^{14} \mathrm{C}$ results so far obtained for the Chauvet-Pont d'Arc Cave are given with an uncertainty of at least $150{ }^{14} \mathrm{C}$ yr, which can grow up to $500{ }^{14} \mathrm{C} \mathrm{yr}$, according to the scarcity of the sample, its weight, etc. (Valladas et al. 2005). The statistical interest of an intercomparison program is in particular to substantially reduce the uncertainty on the sample age. By getting 20 measurements per sample, we succeeded in reducing the uncertainty on the ${ }^{14} \mathrm{C}$ density to $\sim 70{ }^{14} \mathrm{C}$ yr. Thus, this model shows that each time we performed multiple measurements on the same sample, we obtained an average ${ }^{14} \mathrm{C}$ age close to $32 \mathrm{kaBP}$, with a reduced uncertainty. 
Table 2 Results of the First Intercomparison Program carried out on the three ground charcoal specimens GC-40, GC-41, and GC-42. The pretreatment protocols and AMS facilities used are detailed in columns 3 and $4, \delta^{13} \mathrm{C}$ are in column 5. Background corrections applied on each measurement are reported in column $6 .{ }^{14} \mathrm{C}$ ages are reported in $\mathrm{BP}$ (column 7); some laboratories performed multiple dates and their average values are calculated (column 8). $\delta$ average calculates the dispersion of a single date to the total average value (column 9 ). $\delta \sigma$ represents the dispersion of a single date uncertainty to the uncertainty's minimun (column 10). Maxima of $\delta$ average and $\delta \sigma$ are reported at the bottom of columns 9 and 10 for GC-40, GC-41, and GC-42. To identify outliers (WOS), the a priori probability was set to $5 \%$ for each sample's measurements and the a posteriori outlier probabilities were calculated using the OxCal 4.2 R_Combine tool. They are reported in column 11 (prior/posterior).

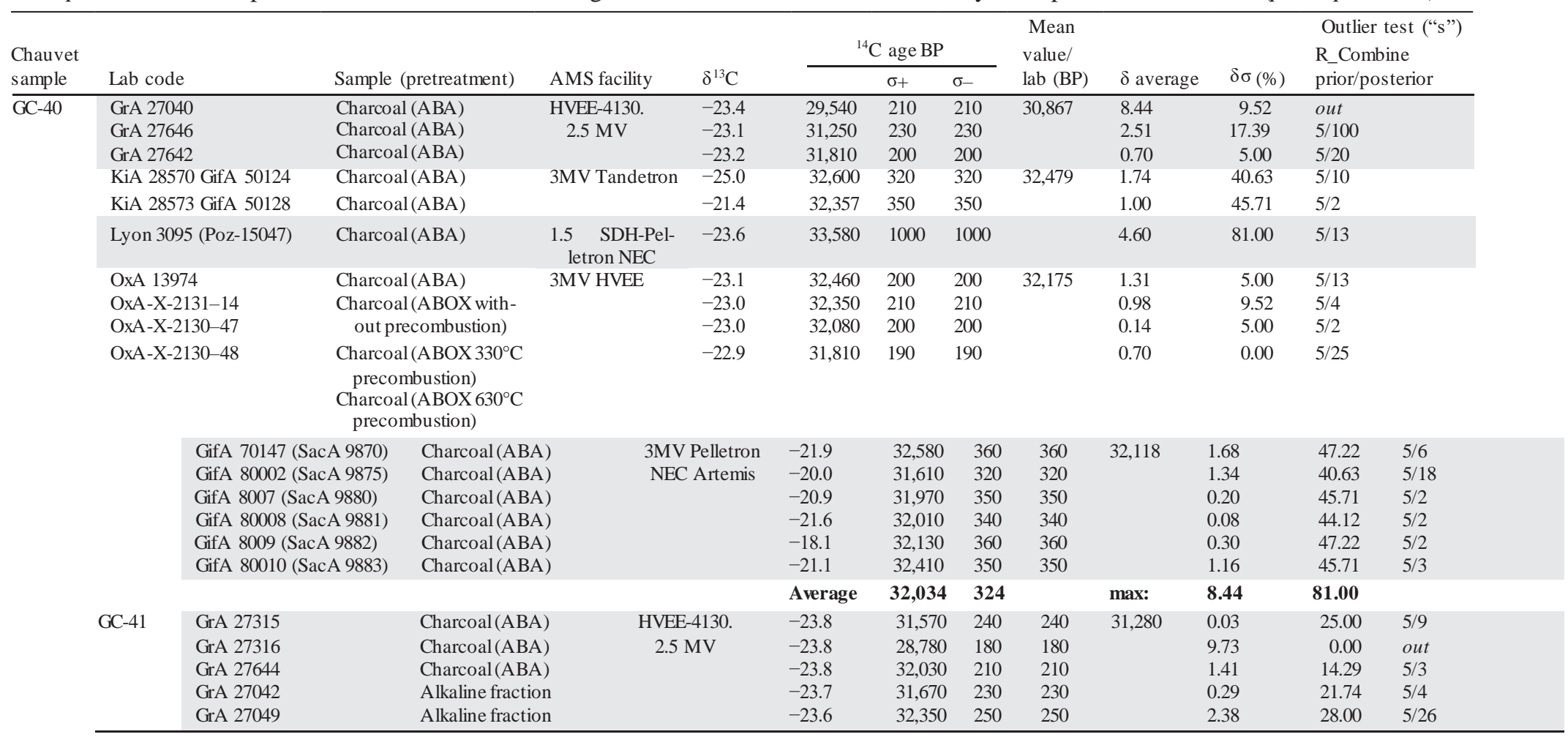


Table 2 (Continued)

\begin{tabular}{|c|c|c|c|c|c|c|c|c|c|c|c|}
\hline $\begin{array}{l}\text { Chauvet } \\
\text { sample }\end{array}$ & Lab code & Sample (pretreatment) & AMS facility & $\delta^{13} \mathrm{C}$ & \multicolumn{3}{|c|}{${ }^{14} \mathrm{C}$ age $\mathrm{BP}$} & $\begin{array}{l}\text { Mean } \\
\text { value/ } \\
\text { lab (BP) }\end{array}$ & $\delta$ average & $\delta \sigma(\%)$ & $\begin{array}{l}\text { Outlier test ("s") } \\
\text { R_Combine } \\
\text { prior/posterior }\end{array}$ \\
\hline & KiA $28574 /$ GifA 50129 & Charcoal(ABA) & $3 \mathrm{MV}$ Tandetron & -23.5 & 32,313 & 310 & 310 & 31,703 & 2.27 & 41.94 & $5 / 9$ \\
\hline & KiA 28595/GifA 50160 & Charcoal(ABA) & & -24.8 & 31,093 & 260 & 260 & & 1.57 & 30.77 & $5 / 94$ \\
\hline & Lyon 3096 (Poz-15048) & Charcoal(ABA) & $\begin{array}{l}1.5 \text { SDH-Pel- } \\
\text { letron NEC }\end{array}$ & -23.6 & 31,590 & 780 & & - & 0.03 & 76.92 & $5 / 2$ \\
\hline & $\begin{array}{l}\text { OxA } 13975 \\
\text { OxA-X-2138-36 }\end{array}$ & $\begin{array}{l}\text { Charcoal(ABA) } \\
\text { Charcoal(ABOX with- } \\
\text { out precombustion) }\end{array}$ & 3MV HVEE & $\begin{array}{l}-23.7 \\
-22.4\end{array}$ & $\begin{array}{l}31,920 \\
31,920\end{array}$ & $\begin{array}{l}180 \\
190\end{array}$ & $\begin{array}{l}180 \\
190\end{array}$ & 31,920 & $\begin{array}{l}1.07 \\
1.07\end{array}$ & $\begin{array}{l}0.00 \\
5.26\end{array}$ & $\begin{array}{l}5 / 2 \\
5 / 2\end{array}$ \\
\hline & $\begin{array}{l}\text { GifA } 70148 \text { (SacA 9871) } \\
\text { GifA } 70054 \text { (SacA 8545) } \\
\text { GifA } 70055 \text { (SacA 8546) } \\
\text { GifA } 80003 \text { (SacA 9876) } \\
\text { GifA } 80174 \text { (SacA 12039) }\end{array}$ & $\begin{array}{l}\text { Charcoal(ABA) } \\
\text { Charcoal(ABA) } \\
\text { Charcoal(ABA) } \\
\text { Charcoal(ABA) } \\
\text { Charcoal(ABA) }\end{array}$ & $\begin{array}{l}\text { 3MV Pelletron } \\
\text { NEC Artemis }\end{array}$ & $\begin{array}{l}-21.0 \\
-18.0 \\
-22.3 \\
-23.7 \\
-24.2\end{array}$ & $\begin{array}{l}32,370 \\
32,100 \\
30,800 \\
31,680 \\
31,510\end{array}$ & $\begin{array}{l}360 \\
360 \\
300 \\
330 \\
270\end{array}$ & $\begin{array}{l}360 \\
360 \\
300 \\
330 \\
270\end{array}$ & 31,692 & $\begin{array}{l}2.44 \\
1.62 \\
2.53 \\
0.32 \\
0.22\end{array}$ & $\begin{array}{l}50.00 \\
50.00 \\
40.00 \\
45.45 \\
33.33\end{array}$ & $\begin{array}{l}5 / 8 \\
5 / 2 \\
5 / 100 \\
5 / 3 \\
5 / 10\end{array}$ \\
\hline & & & & Average : & 31,580 & 297 & & $\max :$ & 9.73 & 76.92 & \\
\hline \multirow[t]{6}{*}{ GC-42 } & $\begin{array}{l}\text { GrA } 27044 \\
\text { GrA } 27045 \\
\text { GrA } 27051 \\
\text { GrA } 27052 \\
\text { GrA } 27645\end{array}$ & $\begin{array}{l}\text { Charcoal(ABA) } \\
\text { Charcoal(ABA) } \\
\text { Charcoal(ABA) } \\
\text { Alkaline Fraction } \\
\text { Alkaline Fraction }\end{array}$ & $\begin{array}{l}\text { HVEE-4130. } \\
2.5 \mathrm{MV}\end{array}$ & $\begin{array}{l}-23.7 \\
-23.5 \\
-23.5 \\
-23.6 \\
-23.6\end{array}$ & $\begin{array}{l}31,960 \\
31,390 \\
31,570 \\
30,290 \\
31,870\end{array}$ & $\begin{array}{l}240 \\
230 \\
300 \\
210 \\
210\end{array}$ & $\begin{array}{l}240 \\
230 \\
300 \\
210 \\
210\end{array}$ & 31,416 & $\begin{array}{l}0.49 \\
1.31 \\
0.73 \\
4.99 \\
0.21\end{array}$ & $\begin{array}{r}16.67 \\
13.04 \\
33.33 \\
4.76 \\
4.76\end{array}$ & $\begin{array}{l}5 / 4 \\
5 / 14 \\
5 / 3 \\
5 / 100 \\
5 / 3\end{array}$ \\
\hline & $\begin{array}{l}\text { KiA 28575/GifA50130a } \\
\text { KiA 28575/GifA50130b }\end{array}$ & $\begin{array}{l}\text { Charcoal(ABA) } \\
\text { Charcoal(ABA) }\end{array}$ & 3MV Tandetron & $\begin{array}{l}-23.9 \\
-23.0\end{array}$ & $\begin{array}{l}32,191 \\
31,700\end{array}$ & $\begin{array}{l}380 \\
280\end{array}$ & $\begin{array}{l}380 \\
280\end{array}$ & 31,946 & $\begin{array}{l}1.21 \\
0.32\end{array}$ & $\begin{array}{l}47.37 \\
28.57\end{array}$ & $\begin{array}{l}5 / 6 \\
5 / 2\end{array}$ \\
\hline & Lyon 3097 (Poz-15049) & Charcoal(ABA) & $\begin{array}{l}1.5 \text { SDH-Pel- } \\
\text { letron NEC }\end{array}$ & -23.1 & 32,200 & 950 & 950 & - & 1.24 & 78.95 & $5 / 2$ \\
\hline & OxA 13976 & Charcoal(ABA) & 3MV HVEE & -22.8 & 32,870 & 200 & 200 & - & 3.25 & 0.00 & $5 / 100$ \\
\hline & $\begin{array}{l}\text { GifA } 70149 \text { (SacA 9872) } \\
\text { GifA } 80004 \text { (SacA 9877) }\end{array}$ & $\begin{array}{l}\text { Charcoal(ABA) } \\
\text { Charcoal(ABA) }\end{array}$ & $\begin{array}{l}\text { 3MV Pelletron } \\
\text { NEC Artemis }\end{array}$ & $\begin{array}{l}-16.8 \\
-20.3\end{array}$ & $\begin{array}{l}32,410 \\
31,370\end{array}$ & $\begin{array}{l}370 \\
320\end{array}$ & $\begin{array}{l}370 \\
320\end{array}$ & 31,890 & $\begin{array}{l}1.88 \\
1.38\end{array}$ & $\begin{array}{r}13.51 \\
0.00\end{array}$ & $\begin{array}{l}5 / 27 \\
5 / 6\end{array}$ \\
\hline & & & & Average : & 31,802 & 335 & & max: & 4.99 & 78.95 & \\
\hline
\end{tabular}




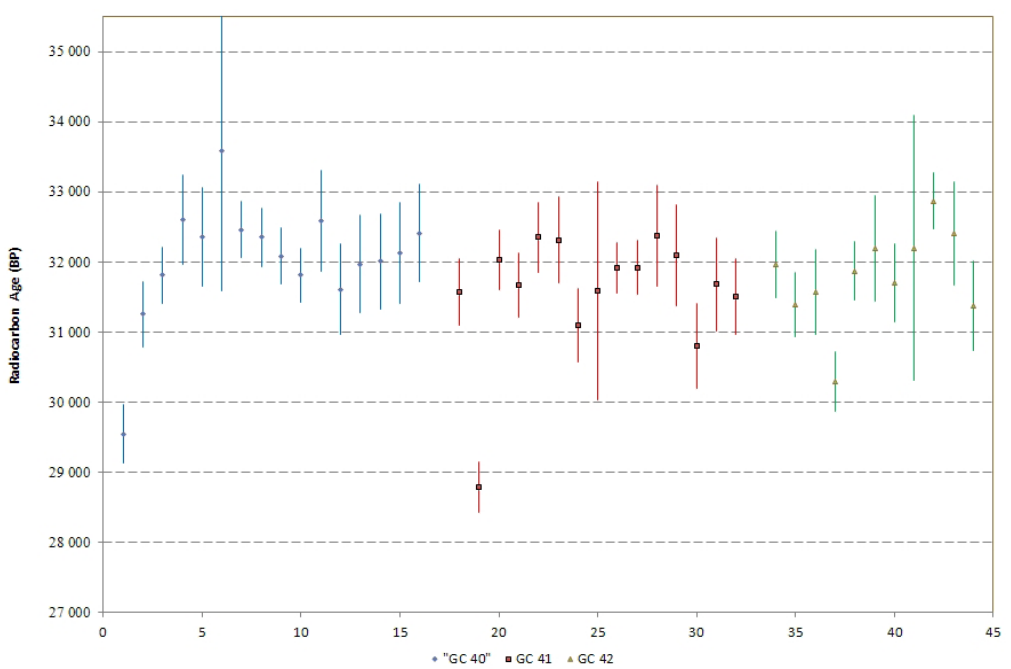

Figure $6{ }^{14} \mathrm{C}$ ages obtained for GC-40 (blue), GC-41 (red) and GC-42 (green), with a $2 \sigma$ range (First Intercomparison Program, Cuzange et al. 2007). The three average ages are respectively set to $32,034 \pm 324,31,580 \pm 297$, and $31,802 \pm 335 \mathrm{BP}$; one measurement per sample is not compatible with this average value with a $2 \sigma$ range.

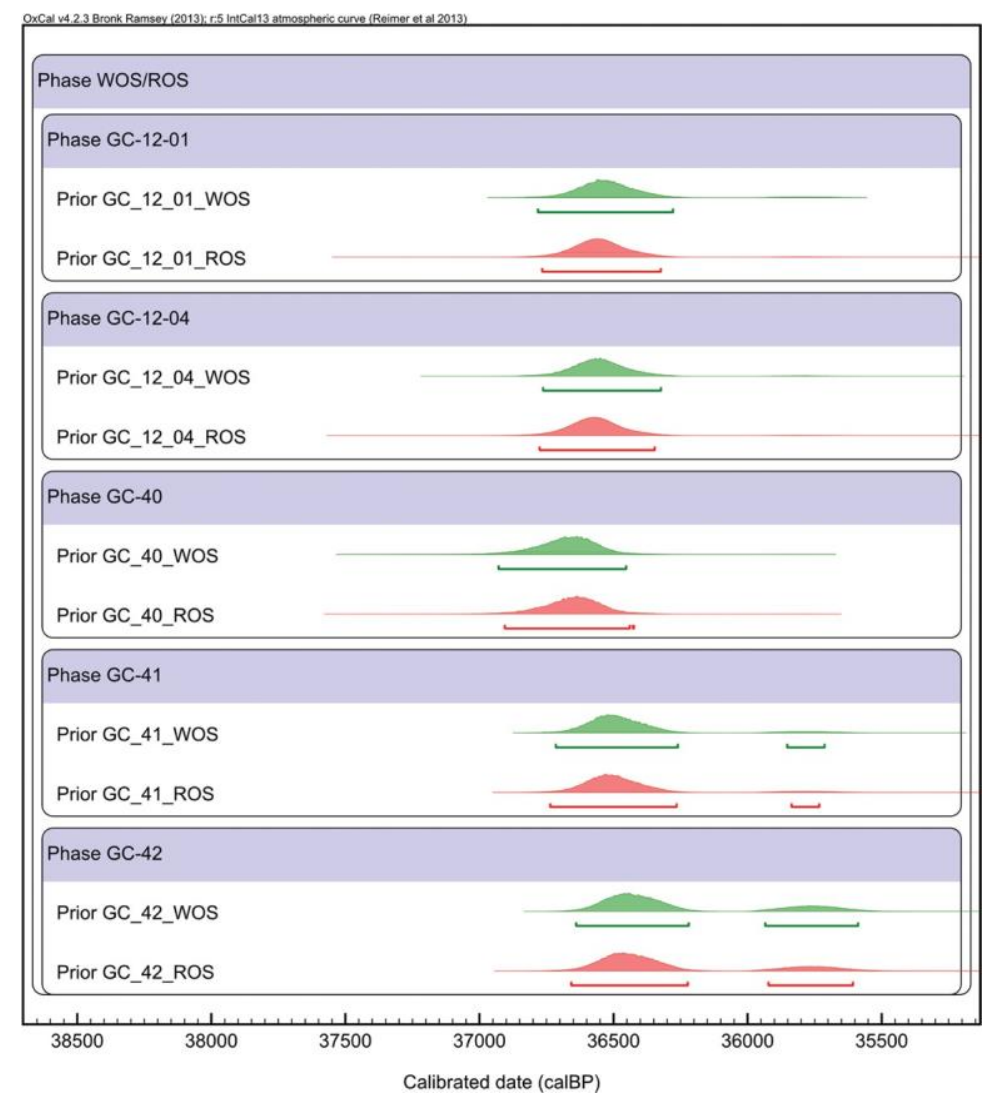

Figure 7 Comparison of calibrated combined densities deduced from the WOS (in green) and the ROS (in red) statistical methods used to deal with outliers. Both WOS and ROS strategies lead to two quasi-identical temporal densities despite the offset between combined ${ }^{14} \mathrm{C}$ ages. 
Table 3 Comparison of the results obtained from the weighted outlier strategy (WOS) and the rejected outlier strategy (ROS). Using the WOS, R_Combine ${ }^{14} \mathrm{C}$ values obtained with an a priori outlier probability set to 5\% for all measurements are reported in column 3 . Their deduced calibrated ranges are given in column 4; the convergence factor $(C)$ is given in column 5 . Results of the $\chi^{2}$ test are given in column 6 . ROS calculation results are reported in columns 7-8. Differences between WOS (column 3) and ROS (column 7) ${ }^{14}$ C results are reported in column 10. We observe an average value of the differences between the two combined ${ }^{14} \mathrm{C}$ ages of $-36{ }^{14} \mathrm{C}$ yr. The length of the calendar intervals between both strategies is presented in column 11. Results of the WOS calculations were selected for the modeling (in green).

\begin{tabular}{|c|c|c|c|c|c|c|c|c|c|c|c|c|c|c|c|c|c|}
\hline \multirow{3}{*}{$\begin{array}{l}\text { Sample } \\
\text { nr }\end{array}$} & \multirow{3}{*}{$\begin{array}{l}\text { Analyses } \\
(n)\end{array}$} & \multicolumn{7}{|c|}{ Weighted outlier strategy (WOS) } & \multicolumn{6}{|c|}{ Rejected outlier strategy (ROS) } & \multirow{3}{*}{$\begin{array}{l}{ }^{14} \mathrm{C} \text { age } \\
\text { difference } \\
{\left[{ }^{14} \mathrm{C} \mathrm{yr}\right]}\end{array}$} & \multirow{2}{*}{\multicolumn{2}{|c|}{$\begin{array}{l}\text { Calendar } \\
\text { interval } \\
(2 \sigma) \quad[\mathrm{yr}]\end{array}$}} \\
\hline & & \multicolumn{2}{|c|}{$\begin{array}{l}\text { R_Combined } \\
\text { (BP) }\end{array}$} & \multicolumn{4}{|c|}{ Calibrated ranges (cal BP) } & \multirow[b]{2}{*}{$\gamma^{2}$} & \multicolumn{2}{|c|}{$\begin{array}{l}\text { R_Combined } \\
\text { (BP) }\end{array}$} & \multicolumn{4}{|c|}{ Calibrated ranges (cal BP) } & & & \\
\hline & & Value & $\sigma$ & $>$ & $<$ & Rang & & & Value & $\sigma$ & $>$ & $<$ & Range & $\gamma^{2}$ & & WOS & ROS \\
\hline GC-12-01 & 21 & 31,843 & 67 & 36,782 & 36,278 & 95.4 & 97 & $\begin{array}{l}48.9 \\
(5 \% 31.4)\end{array}$ & 32,003 & 76 & 36,766 & 36,324 & 95.4 & $\begin{array}{l}24.2 \\
(5 \% 28.9)\end{array}$ & -160 & 504 & $\overline{442}$ \\
\hline GC-12-04 & 22 & 32,078 & 68 & 36,767 & 36,325 & 95.4 & 96 & $\begin{array}{l}34.1 \\
(5 \% 32.7)\end{array}$ & 32,033 & 69 & 36,776 & 36,346 & 95.4 & $\begin{array}{l}26.1 \\
(5 \% 31.4)\end{array}$ & 45 & 440 & 430 \\
\hline GC-40 & 16 & 32,087 & 69 & 36,877 & 36,420 & 95.4 & 98 & $\begin{array}{l}32.316 \\
(5 \% 23.7)\end{array}$ & 32,156 & 72 & 36,866 & 36,429 & 95.4 & $\begin{array}{l}19.5 \\
(5 \% 22.4)\end{array}$ & -69 & 457 & 437 \\
\hline GC-41 & 15 & 31,828 & 70 & 36,724 & 35,719 & 95.4 & 98 & $\begin{array}{l}32.568 \\
(5 \% 22.4)\end{array}$ & 31,875 & 72 & 36,700 & 35,705 & 95.4 & $\begin{array}{l}21.787 \\
(5 \% 21.0)\end{array}$ & -47 & 1005 & 995 \\
\hline GC-42 & 11 & 31,832 & 81 & 36,641 & 35,599 & 95.4 & 98 & $\begin{array}{l}85.789 \\
(5 \% \quad 18.3)\end{array}$ & 31,782 & 97 & 36,652 & 35,610 & 95.4 & $\begin{array}{l}10.2 \\
(5 \% \text { 15.5) } \\
\text { erage diffe }\end{array}$ & 50 & 1042 & 1042 \\
\hline
\end{tabular}




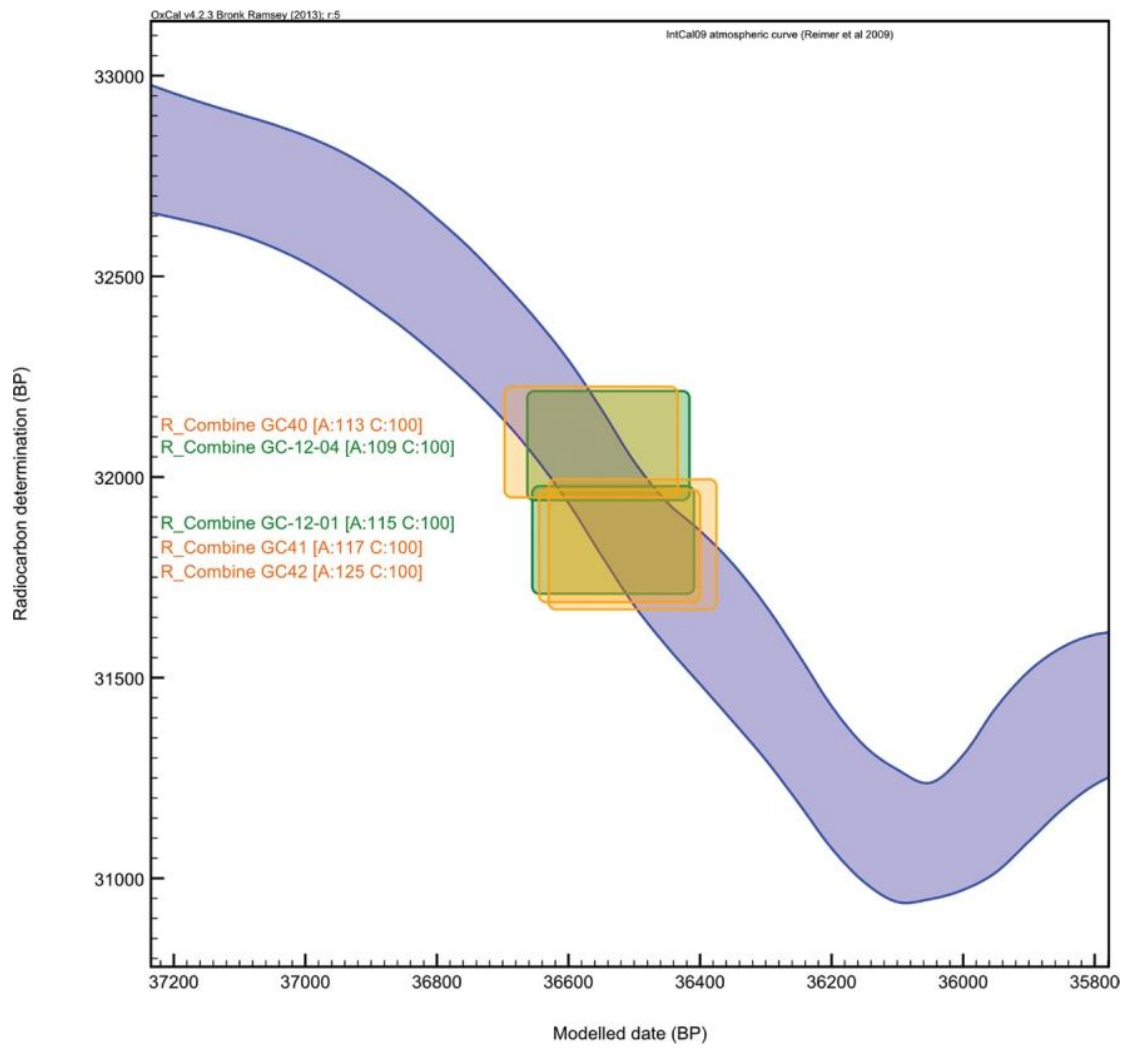

Figure 8 The five combined temporal densities deduced from the two intercomparison programs are linked before the plateau age of the calibration curve (First Intercomparis on Program in yellow, Second Intercomparis on Program in green). They give a calendar age focused on $36.5 \mathrm{ka} \mathrm{cal} \mathrm{BP.} \mathrm{The}$ five densities are associated with a human activity that occurred in the Galerie des Mégacéros and was as sociated with the first human occupation. This occupation phase, sequenced with an older and a younger boundary, occurred sometime between 36.8 and 36.2 ka cal BP $(2 \sigma)$.

As a result, it is truly informative to observe that five different samples from at least three different and independent hearth structures give very similar results. They are associated with one human occupation in the Galerie des Mégacéros. In order to model this occupation phase, we gathered these five combined densities in a phase, which was sequenced with an older and a younger boundary (boundary Start and boundary End) (Bronk Ramsey 2009a). Figure 8 shows the five WOS combined densities obtained on the calibration curve; they are perfectly consistent. The Boundary Start modeled an age extending from 36.8 to $36.4 \mathrm{ka}$ cal BP $(2 \sigma)$ and the Boundary End from 36.6 to $36.2 \mathrm{ka}$ cal BP $(2 \sigma)$ (Figure 8$)$. These two modeled intervals are clearly consistent. Note that this model integrates a set of $85{ }^{14} \mathrm{C}$ dates. This leads to the conclusion that human activity occurred in the Galerie des Mégacéros of the Chauvet-Pont d'Arc Cave between 36.8 and 36.2 ka cal BP $(2 \sigma)$, linked with the first human occupation.

\section{CONCLUSION}

This article reports the results of the Second Intercomparison Program for the Chauvet-Pont d'Arc Cave. Two large charcoal specimens from two independent archaeological hearth structures, sampled in the Galerie des Mégacéros, have been independently dated by 10 laboratories. GC-12-01 resulted in 21 measurements, whereas 22 analyses were performed on GC-12-04. Those two sets 
of ${ }^{14} \mathrm{C}$ dates are consistent. Both average values are close to $32 \mathrm{ka} \mathrm{BP}$ and reach the same average uncertainty $\left(\sim 300{ }^{14} \mathrm{C} \mathrm{yr}\right)$. In terms of average ${ }^{14} \mathrm{C}$ measurements, they are close with a maximum dispersion of $3 \%$. Therefore, analysis reproducibility is confirmed, whate ver the pretreatment protocol and facility used.

To deal with outliers, we tested two different ways to model the rejection of samples. First, we manually rejected ${ }^{14} \mathrm{C}$ measurements that were not consistent with the average value within a $2 \sigma$ range. Then, we used the OxCal Outlier detection model SSimple to identify the outliers. Using the weighted outlier strategy (WOS), we weighted the probability to be an outlier by giving to each measurement an offset in proportion to how likely the sample seemed to be an outlier. Using the rejected outlier strategy (ROS), spurious results getting the highest outliers probabilities were removed manually, one after the other, and the model was rerun until getting a model that validated the $\chi^{2}$ test. We finally deduced one R_Combine density for each strategy and sample. Calibration of these previous densities has shown that these two methods of dealing with outliers lead to the same combined calendar densities.

The intercomparison programs allow for substantial reduction of the uncertainty in the ${ }^{14} \mathrm{C}$ density, and, thus, the calendar range. Results of this Second Intercomparison Program were compared to those of the previous program. The five combined ages show that e very time we succeed in reducing the age uncertainty, we obtain combined ${ }^{14} \mathrm{C}$ densities linked on the calibration curve and close to $32 \mathrm{ka} \mathrm{BP}$. Then, as those five calendar densities (which include $85{ }^{14} \mathrm{C}$ dates) are deduced from the analyses of five different pieces of charcoal coming from three different archaeological entities, it appears reasonable to consider that those densities are associated with a human activity that occurred in the Galerie des Mégacéros sometime between 36.8 and $36.2 \mathrm{ka} \mathrm{cal} \mathrm{BP}(2 \sigma)$ and linked to the first prehistoric occupation within the cave.

\section{ACKNOWLEDGMENTS}

This project has been realized in the framework of the Programme National de la Recherche sur la Connaissance et la Conservation des Matériaux et du Patrimoine Culture (PNRCC) and the Archaeological Research Project for the Chauvet-Pont d'Arc Cave, funded by the French Ministère de la Culture et de la Communication. We are grateful to each of the 10 laboratories, which performed their analyses with their own funding. We are indebted to Isabelle Thery (CEPAM, Nice, France) who performed the anthracological analyses on the two pieces of charcoal.

\section{REFERENCES}

Batten RJ, Gillespie R, Gowlett JAJ, Hedges REM. 1986. The AMS dating of separate fractions in archaeology. Radiocarbon 28(2A):698-701.

Bird MI, Ayliffe LK, Fifield LK, Turney CSM, Cresswell RG, Barrows TT, David B. 1999. Radiocarbon dating of "old" charcoal using a wet oxidation stepped-combustion procedure. Radiocarbon 41(2):127-40.

Brock F, Higham T, Ditchfield P, Bronk Ramsey C. 2010. Current pretreat ment methods for AMS radiocarbon dating at the Oxford Radiocarbon Accelerator Unit (ORAU). Radiocarbon 52(1):103-12.

Bronk Ramsey C. 2009a. Bayesian analys is of radiocarbon dates. Radiocarbon 51(1):337-60.

Bronk Ramsey C. 2009b. Dealing with outliers and offsets in radiocarbon dating. Radiocarbon 51(3):1023-45.
Clottes J, editor. 2001. La Grotte Chauvet - L'art des origines. Paris: Seuil. 224 p.

Clottes J, Chauvet J-M, Brunel-Deschamps E, Hillaire C, Daugas J-P, Arnold M, Cachier H, Ev in J, Fortin P, Oberlin C, Tisnerat N, Valladas H. 1995. Les peintures préhistoriques de la Grotte ChauvetPont-d'Arc (Ardèche, France): datations directes et indirectes par la méthode du radiocarbone. Comptes Rendus de l'Académie des Sciences de Paris 320(série II a):1133-40.

Cuzange M-T, Delqué-Kolic E, Goslar T, Grootes PM, Higham T, Kaltnecker E, Nadeau M-J, Oberlin C, Paterne M, van der Plicht J, Bronk Ramsey C, Valladas H, Clottes J, Geneste J-M. 2007. Radiocarbon intercomparis on program for Chauvet Cave. Radiocarbon 49(2):339-47. 
Geneste J-M, editor. 2003. Recherches pluridisciplinaires dans la grotte Chauvet. Lyon: Journées SPF.

Genty D, Ghaleb B, Plagnes V, Causse C, Valladas H, Blamart D, Massault M, Geneste J-M, Clottes J. 2004. Datations U/Th (TIMS) et ${ }^{14} \mathrm{C}$ (AMS) des stalag mites de la g rotte Chauvet (Ardèche, France): intérêt pour la chronologie des événements naturels et anthropiques de la grotte. Comptes Rendus Palevol 3(8):629-42.

Hajdas I, Bonani G, Furrer H, Mader A, Schoch W. 2007. Radiocarbon chronology of the mammoth site at Niederweningen, Switze rland: results from dating bones, teeth, wood, and peat. Quaternary International 164-165:98-105.

Quiles A, Valladas H, Geneste J-M, Bocherens H, Genty D, Elalouf J-M, Sadier B. 2012. Bayesian modelling of the Chauvet Cave dating. Oral communication presented at the 21st International Radiocarbon Conference, 13 July 2012, Paris, France.

Reimer PJ, Baillie MGL, Bard E, Bayliss A, Beck JW, Blackwell PG, Bronk Ramsey C, Buck CE, Burr GS, Edwards RL, Friedrich M, Grootes PM, Guilderson TP, Hajdas I, Heaton TJ, Hogg A G, Hughen KA, Kaiser KF, Kromer B, McCormac FG, Manning
SW, Reimer RW, Richards DA, Southon JR, Talamo S, Turney CSM, van der Plicht J, Weyhenmeyer CE. 2009. IntCal09 and Marine09 radiocarbon age calibration curves, 0-50,000 years cal BP. Radiocarbon 51(4):1111-50.

Sadier B, Delannoy JJ, Benedetti L, Bourlès DL, Jaillet S, Geneste J-M, Lebatard A-E, Arnold M. 2012. Further constraints on the Chauvet cave artwork elaboration. Proceedings of the National Academy of Sciences of the USA 109(21):8002-6.

Scott EM. 2003. Section 10: summary and conclusions. Radiocarbon 45(2):285-90.

Valladas H, Clottes J, Geneste J-M, Garcia MA, Arnold M, Cachier H, Tisnérat-Laborde N. 2001. Palaeolithic paintings: evolution of prehistoric cave art. Nature 413(6855):479.

Valladas H, Clottes J, Geneste J-M. 2004. Chauvet, la grotte ornée la mieux datée du monde, Dossier Le Temps des Datations. Pour la Science 42:82-7.

Valladas H, Tisnerat-Laborde N, Cachier H, Kaltnecker E, Arnold M, Oberlin C, Evin J. 2005. Bilan des datations carbone 14 effectuées sur des charbons de bois de la grotte Chauvet. Bulletin de la Société Française de Préhistoire 102(1):109-13 
